\title{
Participatory Design of Technologies to Support Recovery from Substance Use Disorders
}

\author{
ZACHARY SCHMITT, University of Minnesota, USA \\ SVETLANA YAROSH, University of Minnesota, USA
}

\begin{abstract}
Substance use disorders, such as alcoholism and drug addiction, are a widespread and hazardous public health issue. Technology designed for the needs and values of people in recovery may be able to supplement traditional treatment options, enhance long-term abstinence maintenance, and create new opportunities for social support. We conducted a series of participatory design workshops with women in recovery from substance use disorders to identify design opportunities for supportive technologies that align with the specific values, practices and traditions of the recovery community. Through a data-driven inductive qualitative analysis, we identify five major themes that may be addressed with technology: 1) supporting twelve-step traditions and practices, 2) management of restlessness and moments of crisis, 3) agency and control over privacy and personal safety, 4) tracking progress and maintaining motivation, and 5) constructing a new normal. We connect these themes to specific implications for design.
\end{abstract}

CCS Concepts: Human-centered computing $\rightarrow$ Collaborative and social computing $\rightarrow$ Empirical studies in collaborative and social computing

\section{KEYWORDS}

Participatory design; Substance use disorders; 12-steps; Peer health support; Technology for mental health

\section{ACM Reference format:}

Zachary Schmitt and Svetlana Yarosh. 2018. Participatory Design of Technologies to Support Recovery from Substance Use Disorders. In Proceedings of the ACM on Human-Computer Interaction, Vol. 2, CSCW, Article 156 (November 2018). ACM, New York, NY. 27 pages. https://doi.org/10.1145/3274425

\section{INTRODUCTION}

Substance use disorders (SUDs), such as alcoholism and drug abuse, represent one of the most widespread and hazardous public health issues facing our society today. In 2015, 20.8 million people aged 12 or older in the United States had a SUD [102]. Each year, SUDs cost the US economy a combined $\$ 442$ billion in expenses related to health care, crime, and lost productivity [102]. For the individual, SUDs can contribute to social, familial and financial difficulties, health complications, and death. Due in part to a dramatic rise in opioid abuse, overdose deaths resulting from SUDs have nearly tripled over the last 20 years [42].

Given the sudden rise in opioid related overdose deaths, the need for effective SUD treatment is great. However, SUDs have been historically difficult to treat given their chronic nature consisting of multiple cycles of treatment, abstinence, and relapse [76]. Previous studies indicate that up to $75 \%$ of individuals relapse within one year following acute SUD treatment (e.g., detoxification and rehabilitation) $[22,33,65,67]$. Consequently, when an individual completes acute treatment, healthcare providers typically recommend a long-term continuing care program, such

Permission to make digital or hard copies of all or part of this work for personal or classroom use is granted without fee provided that copies are not made or distributed for profit or commercial advantage and that copies bear this notice and the full citation on the first page. Copyrights for components of this work owned by others than ACM must be honored. Abstracting with credit is permitted. To copy otherwise, or republish, to post on servers or to redistribute to lists, requires prior specific permission and/or a fee. Request permissions from Permissions@acm.org.

(c) 2018 Association for Computing Machinery ACM 2018 2573-0142/2018/November - ART156 \$15.00 https://doi.org/10.1145/3274425 
as a 12-step group or cognitive behavioral therapy (CBT), to reduce the ongoing risk of relapse [54]. Prior studies indicate that long-term continuing care has a positive impact on SUD outcomes when paired with acute treatment [10], and is considered an essential component of a robust recovery [76].

Despite widespread agreement on the need for long-term continuing care in treating SUDs, many individuals struggle to make a successful transition from acute in-patient treatment to longterm and self-driven continuing care. Many encounter barriers that prevent engagement with traditional continuing care programs such as a lack of knowledge about SUD care options, low expectations of treatment efficacy, concerns about stigma, and a lack of available and flexible treatment options [18,64]. Additionally, those who do engage in long-term continuing care programs often drop-out before the recommended duration [33,51]. Further, even among those who adhere to a long-term continuing care program, the possibility of relapse remains high. Kleber et al. [54] state that social influences (e.g., substance-using family or friends), economic influences (e.g., unemployment), medical conditions (e.g., chronic pain, fatigue), and psychological influences (e.g., hopelessness, despair) increase vulnerability to relapse, even amongst individuals adhering to a continuing care program. Due to the struggles related to traditional forms of long-term continuing care, new and supplemental approaches are needed to extend the impact and accessibility of these potentially lifesaving forms of ongoing support.

Technology may be a promising source of additional support for individuals attempting to make the difficult transition from acute treatment to long-term recovery due to the high penetration of certain technologies (e.g., in 2018, 77\% of Americans owned smartphones [101]), the relatively low cost of scaling (compared to formal treatment interventions), and the potential for customization and personalization. However, prior research indicates the design of such technologies must consider how these systems can complement, rather than replace or contradict, the practices, values, and traditions of existing approaches to recovery [98]. This kind of valuesensitive design is best conducted in close collaboration with relevant participants [13]. In this paper, we describe the process and output of six participatory design workshops conducted with women residents of a sober living home to explore how technology may enhance and compliment traditional forms of long-term continuing care. Through this approach, we address three research questions:

RQ1. What specific challenges of early recovery may be ameliorated by supportive technologies?

RQ2. What current recovery practices and behaviors may benefit most from supportive technologies?

RQ3. What specific values and traditions should guide supportive technologies in this domain?

We begin by providing brief background on current approaches and important terms related to SUD recovery. We connect our work with the previous investigations of technologies for mental health and relevant methodological approaches in the field. We describe our participatory design method and discuss themes resulting from the qualitative analysis of our design workshops. Finally, we connect our findings with broader implications for design in this critical context.

\section{BACKROUND: RECOVERY FROM SUDS}

The following terms and concepts are important for understanding the experience of people in early recovery from SUDs: 
- Treatment: Analogous to treatment models for other chronic conditions, treatment for individuals with SUDs occurs in temporal phases including initial assessment, acute stabilization, and long-term continuing care [54]. Individuals with SUDs require an individualized medical assessment at the onset of treatment to determine immediate physical and psychological risks and to develop an overall treatment plan. Following assessment, individuals undergo detoxification. For cases with a high risk of medically complicated withdrawal symptoms, detoxification often occurs in an inpatient facility under the supervision of a medical professional. Due to the high rate of chronic relapse for individuals with SUDs, relapse prevention programs are essential for long-term recovery. With very few exceptions, individuals in treatment for SUDs are referred to long-term continuing care programs such as 12-step groups or formal evidence-based treatments such as cognitive behavioral therapy (CBT) [63]. Depending on the treatment plan, continuing care may be paired with relapse prevention medications (e.g. Naltrexone) to prevent the pleasurable effects of substance use (e.g. pain relief and feelings of wellbeing) and reduce cravings [54].

- Relapse: While harm reduction approaches are becoming more common, most treatment programs advocate participants to strive towards complete abstinence from all recreational drugs and alcohol. Individuals usually track their continuous abstinence as "sober" or "clean" time and a return to substance use is considered a relapse that resets this clock. The majority of patients who are treated for SUDs have at least one relapse during the first year following treatment [54].

- Role of the 12-step Approach in Long-Term Continuing Care: Despite the recent increased focus on formal evidence-based care (e.g. CBT), 12-step groups such as Alcoholics Anonymous (AA) and Narcotics Anonymous (NA) are the most commonly used form of long-term continuing care program among individuals with SUDs in the United States [36,37]. The prevalence of the 12-step approach in SUD continuing care is due in part to its wide reaching availability [54], free cost of membership, and the high rate of referrals from professional treatment centers [86]. Prior research indicates that up to $80 \%$ of treatment centers refer patients to 12 -step groups following acute treatment $[45,50,52]$.

- Sponsorship, Stepwork, Meetings, and Service: four specific activities espoused by 12step groups as components of a strong recovery program. Sponsorship involves working with a member of the program who has more experience in recovery. The nature of this relationship focuses on the sponsor guiding their sponsee through the 12-steps. Such stepwork usually consists of sharing written journal entries on topics such as admitting the problem, understanding one's maladaptive behaviors or responses, making amends to others, and establishing spiritual maintenance practices. Both 12-step groups and alternative programs (e.g., SMART recovery [44]), emphasize the importance of regular in-person attendance and sharing at social support meetings. Early in recovery, daily meeting attendance is encouraged. Finally, 12-step groups also encourage involvement in service activities such as participating in outreach meetings at hospitals and institutions, helping with the logistics of running a meeting, and participating at the regional and national levels of a 12-step group.

- Sober Living Environment (SLE): is intended to serve as a transitional residence for individuals no longer enrolled in acute treatment who are unable to manage living 
independently without significant risk of relapse [54]. SLEs are alcohol and drug-free living atmospheres that typically require residents to comply with house rules such as maintaining abstinence, paying rent and other fees, participating in house chores, and attending house meetings [75]. Furthermore, SLEs typically mandate or encourage residents to participate in 12-step groups [75]. Even among SLEs that do not mandate 12step participation, residents are likely to participate in 12-step activities due to social norms and the availability of these programs (e.g., [69,75]). In this study, we worked with an SLE that is part of the Minnesota Association of Sober Homes (MASH). All 150 sober homes associated with MASH require residents to meet with a 12-step sponsor and attend three 12-step meetings per week. While this requirement is common nationwide, we discuss this particular perspective throughout this paper.

Finally, in this paper, we do not seek to advocate for a specific treatment approach or maintenance program, rather we seek to connect with and design towards the lived experience, priorities, and values of people in early recovery from SUDs with the goal of amplifying their voices in the design process.

\section{RELATED WORK}

In this section, we review relevant work across HCI, Social Computing, and clinical domains. We articulate the importance of user-centered design and contrast it with current approaches in designing for SUD recovery. Based on previous work, we propose participatory design as a compelling method for user-centered design for SUD recovery.

\subsection{Technology Design for SUDs as a Mental Health Disorder}

As SUDs are one example of a mental health disorder (as designated by the DSM-5 [5]), this study can be informed by designs for mental health. HCI has a long tradition in working with individuals with mental health disorders to co-design user-centered technology to support treatment and ongoing care. However, a similar design emphasis for SUD recovery has rarely been investigated, despite the great potential for user-centered technology to support individuals in managing the immense emotional, physical, and environmental demands of SUD recovery.

3.1.1 HCI Technology for Mental Health. Recently, medical literature has stressed the importance of enabling patients to take an active role in managing their care [11], especially with regard to chronic conditions that require treatment beyond acute in-patient interventions $[35,60]$. Similarly, recent HCI design research has focused on supporting the user's ability to self-monitor and take an active role in the management and treatment of chronic mental health conditions such as depression [26,66], stress [55,59,73,74] general anxiety [96], bipolar disorder [8,9,62], mental illness [70], and ADHD [24]. In addition to developing technology on the behalf of individuals, many HCI studies have co-designed technology with mental health practitioners $[61,94]$ and with individuals with mental health disorders [62,70,85,94]. For example, Matthews et al. [62] conducted a participatory design with nine individuals diagnosed with bipolar disorder to co-design MoodRythm, a mobile app that helps users set and track their daily routines. Using a similar approach, Simm et al. [85] co-developed wearable technology for individuals diagnosed with autism to assist in anxiety self-management.

3.1.2 HCI Technology for SUDs. In contrast, HCI design research regarding SUDs has been qualitatively different from the user-centered approach adopted for other areas of mental health. 
The majority of HCI research regarding SUDs has focused on detecting substance use, rather than providing digital tools to support self-management of the emotional, physical, and environmental factors critical to long-term recovery. Such designs have included mobile phone enabled sensors that detect and track substance use through analysis of breath [95], saliva [100], and walking gait [47]. Additionally, research has also utilized social media language and behavior to categorize and predict relapse and recovery $[58,87,88]$. Furthermore, a small number of studies have focused on the evaluation of commercially made technologies (e.g. [79]). However, the majority of these commercially made technologies have not been examined in peer-reviewed work nor has their design been developed in a transparent manner.

None of the aforementioned designs were developed through formative research nor collaboration with people in recovery from SUDs, despite recent qualitative investigations that suggest the unique perspectives of people in recovery need to be considered to design technologies that are sensitive to the values and priorities of long-term recovery $[80,98]$.

\subsection{Clinical Technology Design for SUDs}

Outside of HCI, the majority of evidence-based supportive technology for SUDs has been developed in clinical domains. Many of these technologies were designed using a top-down approach originating from theoretical perspectives [32] or the role of the clinician [16,41], rather than a patient-driven bottom-up approach. For instance, Gustafson et al. [38,39] based their design of ACHESS, a smartphone platform created to improved long-term recovery of individuals with an Alcohol Use Disorder (AUD), on the principles of self-determination theory. Primary care physicians and individuals with an AUD completed a needs assessment survey and reacted to technological components of ACHESS, but were not directly involved in its design. Similarly, Dulin et al. [27] based the design of their SUD recovery smartphone platform (LBMI-A) on theoretical perspectives of behavioral change including motivation enhancement, relapse prevention, and community reinforcement. Researchers consulted individuals with an AUD in the final design stage, but they were not directly involved in the initial design of LBMI-A. As examples of cliniciandriven design, Carrol et al. [16] and Hasin et al. [41] studied the clinical efficacy of pairing computerized behavioral interventions with treatment-as-usual therapy in an attempt to extend the role of the therapist beyond in-person sessions.

A clear limitation of previous research regarding supportive technologies for SUDs is a lack of design work with people in recovery to seek alignment with the specific priorities, values, and challenges faced by individuals as they transition from acute in-patient treatment to long-term independent recovery. In this study, we attempt to address this gap by using a participatory design approach that positions individuals in early recovery from SUDs as co-designers and subject matter experts in the design of supportive technology.

\subsection{Participatory Design}

Participatory design (PD) was developed on the belief that those that are most affected by the introduction of a new technology have the right to participate in its creation. PD is a widely used approach within the HCI community that positions the end-user as a full participant in the design process [68]. This method is particularly salient when the goal is to empower traditionally marginalized or overlooked user groups by providing them a voice in the design process [68]. For example, Azenkot et al. [6] worked in concert with individuals with visual impairments to design service robots to assist in guiding the blind. Similarly, in their design of motivational systems for 
stroke rehabilitation, Balaam et al. [7] worked directly with individuals in recovery from stroke. Researchers have also adopted the PD approach to co-design technology with individuals with special needs [34], children [99], elderly adults [21,92,93], and individuals with dementia [57]. Given its saliency in empowering traditionally marginalized populations, PD may be particularly appropriate for the female participants in this study. Prior studies indicate women are an often overlooked population within traditional SUD research [91].

In addition to empowering users, $\mathrm{PD}$ is effective in merging the tacit knowledge areas of both designers and users [28]. The knowledge gap positioned between the expert domains of the designer and user is a fertile environment in which participants can combine their diverse knowledge bases to challenge assumptions and develop novel insights [68]. Findings from previous research investigating the use of technology to support SUD recovery strongly suggest that design should complement, rather than replace or contradict, the practices, values, and traditions of existing approaches to recovery [98]. Thus, in this study, we utilize the participatory design approach to position participants as co-designers and subject matter experts to ascertain how technology can supplement, rather than disrupt, their recovery efforts.

\section{METHODS}

In order to align the design of supportive technology with the values and priorities of the recovery community, we conducted six participatory design workshops with women in early recovery from SUDs. During these workshops, participants discussed how and why they currently use technology to support their recovery, interacted with currently available technology designed for SUD recovery, identified prominent problems related to their recovery, brainstormed technical solutions to these problems, and finally sketched the most promising solutions.

\subsection{Ethical Considerations}

Recovery from substance use is a demanding and private endeavor, requiring additional consideration of participant protections. We took several steps to safeguard participant anonymity and well-being during the study. We wanted to ensure that the identity of each participant remained anonymous even if one of them chose to disclose their own participation to others outside of this study (this action would reveal our partner SLE and could therefore allow others to identify other participants based on demographic data). To do so, we chose not to collect participant demographic data (e.g., age, length of sobriety, substance use history) that could point to a participant's identity. Furthermore, we sought and received permission from the IRB to waive the documentation of consent. We handled compensation for participation in each workshop through anonymous gift cards handed out at the end of each workshop, thus precluding the need for participants to contact us to receive pro-rated compensation if they could not participate in future workshops. Additionally, through a special dispensation from our department, we did not collect names or signatures to confirm compensation. By removing the requirements for documenting consent and compensation, we circumvented the need for participants to provide their name at any point in the process.

In addition to safeguarding participant confidentiality, we took steps to ensure that research workshops did not interfere with activities beneficial to participant recovery. We worked with SLE managers and residents to schedule the participatory design workshops around events related to participant recovery including AA or NA meetings, meeting with sponsors, job duties, and service 
commitments. Individuals could self-select to participate (or not participate) in the workshops that fit in their schedule on a case-by-case basis.

\subsection{Research Site and Participants}

We worked with residents of a women's SLE. The SLE provides a safe and drug-free residence where women lend support to each other as they begin the long-term process of SUD recovery. Generally, residents are in early recovery (e.g., $<1$ year clean/sober) as SLE residency serves as a stepping-stone to independent living.

As part of their decision to live in the SLE, residents agreed to a number of house rules intended to provide structure and support to their recovery. Primarily, residents were required to attend three 12-step group meetings per week, meet with a sponsor, and engage in service work (the majority of SLE's nationwide either require or strongly encourage 12-step participation [75]). Additionally, residents were required to spend a minimum of 25 hours per week working, attending school, or attending recovery related activities. Residents were free to choose the specific type of 12 -step program in which they were to participate. For example, the SLE manager informed us that some residents attended 12-step groups that emphasized mindfulness meditation and others participated in 12-step groups for non-believers (i.e. atheist 12-steps). Furthermore, residents could supplement their participation in 12-step groups with other forms of treatment such as CBT, SMART recovery, etc.

At the time of the study, sixteen women resided in the SLE and each of them took part in at least one participatory design workshop (of which there were six). Most residents attended multiple workshops and some participated in all workshops (see Table 1 for workshop attendance). As previously stated, to protect the anonymity of the participants, we did not collect individualized demographic data. However, the SLE manager provided aggregate information such as the average age of residents (29.5 years old), the average length of stay (10 months), and the specific substances used by residents which included alcohol, heroin and other opioids, methamphetamine, ketamine, cocaine, MDMA (commonly known as ecstasy), and benzodiazepine. Some participants were in recovery from the use of multiple substances. Each participatory design workshop took place in the living room of the SLE.

\subsection{Procedure}

As not to interfere with the participants' recovery related activities, the SLE manager recommended that the duration of our interactions with them be restricted to a maximum of ninety minutes per session. Therefore, rather than conducting a single participatory design workshop that would have engaged participants for many hours, we conducted six separate ninety-minute workshops that took place over the course of six weeks (see Table 1 for more information). Each workshop was separated by an interval of five to ten days. The divided structure was also beneficial to us as researchers. We used the data collected in each workshop to prepare for and adjust the content of subsequent workshops to create a more engaging and productive experience for participants. For example, after the initial brainstorming and idea selection workshop, the researchers tallied votes to present only the most promising ideas to the group for the following session.

In the first participatory design workshop, participants discussed their experience using technology to support their recovery. Participants formed groups of three to discuss and record (on poster paper) the names of the technologies they used to support their recovery, how it 
supported their recovery, as well as the device they used to access said technology. Following small group discussion, each group presented their poster.

In the second workshop, participants interacted with commercially made smartphone applications designed specifically to support SUD recovery (see Table 1). We selected popular applications, many of which were not currently used by participants, to expose them to the breadth of supportive technologies currently available. Participants spent 15-20 minutes exploring each app and then recorded what they liked and disliked about each app. Participants followed the same protocol in workshop 3, but interacted with smartphone applications designed for the promotion

\begin{tabular}{|c|c|c|c|}
\hline Workshop & $\mathbf{N}$ & Participant Activities & Engagement \& Research Goals \\
\hline $\begin{array}{l}\text { 1. Currently } \\
\text { Used Tech } \\
\text { Workshop }\end{array}$ & 10 & $\begin{array}{l}\text { Listed and discussed the } \\
\text { technology they use to support } \\
\text { their recovery. }\end{array}$ & $\begin{array}{l}\text { - Gather in-depth accounts of how and why } \\
\text { participants currently use technology for } \\
\text { recovery. } \\
\text { - Determine participant familiarity with } \\
\text { technology to inform upcoming workshops. }\end{array}$ \\
\hline $\begin{array}{l}\text { 2. Recovery } \\
\text { App } \\
\text { Workshop }\end{array}$ & 5 & $\begin{array}{l}\text { Interacted with and reflected on } \\
\text { four apps designed to support } \\
\text { recovery: WeConnect }{ }^{1} \text {, } \\
\text { Addicaid }^{2} \text {, InTheRooms }{ }^{3} \text {, and } \\
\text { NoMo }^{4} \text {. }\end{array}$ & $\begin{array}{l}\text { - Prepare participants for upcoming design } \\
\text { workshops by demonstrating existing SUD } \\
\text { recovery apps. } \\
\text { - Gather participant impressions of each app. } \\
\text { - Identify technologies that may aid in their } \\
\text { recovery. }\end{array}$ \\
\hline $\begin{array}{l}\text { 3. Health } \\
\text { Promotion } \\
\text { App } \\
\text { Workshop }\end{array}$ & 3 & $\begin{array}{l}\text { Interacted with three health apps } \\
\text { not specifically designed for SUD } \\
\text { recovery }\left(\mathrm{KoKo}^{5}, \text { Pacifica }^{6} \text {, and }\right. \\
\left.\text { Fitbit }^{7}\right) \text { and reflected on the } \\
\text { applicability to recovery. }\end{array}$ & $\begin{array}{l}\text { - Prepare participants for upcoming design } \\
\text { workshops by demonstrating existing health } \\
\text { apps. } \\
\text { - Gather participant impressions of each app. } \\
\text { - Identify technologies that may aid in their } \\
\text { recovery. }\end{array}$ \\
\hline $\begin{array}{l}\text { 4. Problem } \\
\text { Identification } \\
\text { Workshop }\end{array}$ & 9 & $\begin{array}{l}\text { Identified twenty-five problems } \\
\text { related to their SUD recovery and } \\
\text { voted for the problems they } \\
\text { found most personally relevant. }\end{array}$ & $\begin{array}{l}\text { - Identify core themes in the most prominent } \\
\text { problems participants face in day-to-day } \\
\text { recovery. } \\
\text { - Provide focus and context for the upcoming } \\
\text { ideation and sketching workshops. }\end{array}$ \\
\hline $\begin{array}{l}\text { 5. Ideation } \\
\text { Workshop }\end{array}$ & 7 & $\begin{array}{l}\text { Brainstormed fifty-four potential } \\
\text { technical solutions to problems } \\
\text { identified in workshop \#4, and } \\
\text { voted for the most promising } \\
\text { solutions. }\end{array}$ & $\begin{array}{l}\text { - Teach participants IDEO-style }{ }^{8} \text { ideation } \\
\text { techniques. } \\
\text { - Brainstorm at least } 50 \text { potential } \\
\text { technological solutions to the problems } \\
\text { outlined in workshop \#4. } \\
\text { - Identify core themes in participant ideation. }\end{array}$ \\
\hline $\begin{array}{l}\text { 6. Sketching } \\
\text { Workshop }\end{array}$ & 8 & $\begin{array}{l}\text { Worked in groups to sketch their } \\
\text { favorite ideas from workshop \#5. }\end{array}$ & $\begin{array}{l}\text { - Provide participants with agency in } \\
\text { designing technology that will benefit them } \\
\text { most. } \\
\text { - Identify core themes in participant designs. }\end{array}$ \\
\hline
\end{tabular}

Table 1. Study procedure including the number of participants, activities, and goals for each participatory design workshop.

\footnotetext{
${ }^{1}$ https://weconnectrecovery.com/

${ }^{2}$ https://addicaid.com/

${ }^{3}$ https://www.intherooms.com/

${ }^{4} \mathrm{http}: / /$ meetnomo.com/

${ }^{5}$ https://itskoko.com/

${ }^{6}$ http://www.thinkpacifica.com/

${ }^{7}$ https://www.fitbit.com

${ }^{8}$ http://www.designkit.org/methods/28
} 
of emotional and physical health (e.g. Fitbit, Koko, Pacifica), rather than apps designed specifically for SUD recovery.

We designed the first three workshops to accomplish three core objectives towards familiarizing participants with supportive technology for recovery including: 1) expose participants to supportive technology that may aid them in their personal SUD recovery process, 2) demonstrate technical possibilities of supportive technology to better prepare participants to brainstorm and design their own solutions in upcoming workshops, and 3) identify gaps in the current landscape of supportive technology for SUD recovery. During the subsequent and final three workshops, we utilized the technological foundation we built with participants over the first three workshops to identify prominent recovery challenges and to brainstorm and design technological solutions to these challenges.

During the fourth workshop, participants identified the most prominent and difficult problems they face in their day-to-day recovery. We asked participants to give specific attention to the problems that currently do not have an effective technological solution or technological support system. Framing the workshop in this manner allowed us to challenge participants to focus on aspects of their recovery not supported by the current landscape of supportive SUD technologies. Additionally, the results from this workshop allowed us to shape the agenda of the upcoming brainstorming workshop to focus on problems that were personally relevant to the participants. Thus, in the fifth workshop (i.e., brainstorming workshop), we revisited the problems that participants deemed most personally relevant and worked with them to brainstorm technological solutions to these problems (see Table 1 for more detail).

In the sixth and final workshop, participants worked in groups of two or three to sketch designs for supportive technologies inspired by the technological solutions they developed in the preceding workshop. To attenuate the impact of the relatively low attendance of the second the third workshops (see Table 1), we ensured that a participant that attended either the second or the third workshop was present in each sketch group. Additionally, we reviewed the main findings of previous workshops at the start of each subsequent workshop to contextualize the activity for participants.

\subsection{Analysis}

During the participatory design workshops, we collected qualitative data including researcher field notes (as a team of researchers carried out the work, one member could lead the workshops while the other took detailed notes), participant discussions, lists of problems related to recovery, participant writing, brainstormed ideas, and participant design artifacts (e.g. sketches). To protect participant anonymity, we did not use audio or video recording. We converted non-textual data (e.g., reflections on field notes, descriptions of participant design artifacts) to text through the generation of memos when appropriate. We analyzed this textual dataset using data-driven inductive thematic analysis characterized by the generation and constant comparison of open codes in order to reveal underlying themes (e.g., [71]). We began the analysis by performing line-by-line open coding on the qualitative data collected from each workshop, identifying short, individual units of meaning within the textual data. We used the process of affinity diagramming to spatially cluster open codes together based on similar meaning. We discussed and iteratively refined these clusters to identify core themes in the data. In this paper, we report emerging themes that are relevant to opportunities, challenges, and considerations for the design of SUD recovery technologies. 


\subsection{Study Limitations}

While the participatory design method allowed us to incorporate the experience, values, and priorities of individuals in recovery from SUDs into the design of supportive technology, the methodology contains inherent limitations. Primarily, participatory design is a subjective process and carries with it the individual perspectives of both participants and researchers. A different group of participants or researchers may have come to different conclusions, focused on different obstacles related to recovery, and may have emphasized different design solutions. These limitations are true of all qualitative work and future studies should triangulate with work that utilize other methods.

Furthermore, the small size and homogeneity of our participant group limits the external validity of our findings. Participants in our study were exclusively women residents of an SLE, the majority of which were young (mean age of 29.5). Previous research on gender differences in SUDs suggests that men and women differ in the onset of substance use, social stigmas resulting from substance use, and barriers to treatment [14]. Furthermore, young individuals who voluntarily enter an SLE may have different needs and priorities compared to older individuals, those who are no longer living in an SLE, or those who have never lived in an SLE. Additionally, participants were required to participate in 12-step groups as part of their living agreement with their SLE. While this mandate is very common among SLE's nationwide, and 12-step groups are the most common help setting for adults with SUDs, the requirement limits the generalizability of our results. Future research should seek to extend our findings by engaging in co-design activities with a more diverse or a complementary set of participants.

Despite these limitations, we believe our work provides valuable insight into the design of supportive technology for SUD recovery by establishing alignment with the values, traditions, and priorities of the recovery community. Furthermore, while the homogeneity of our participant group limits generalizability, we believe it is also a strength of this paper. Our specific context and setting enabled us to the focus on the unique experience and needs of women in recovery - an often overlooked population within SUD research [91].

\section{RESULTS}

Through our analysis, we identified 5 major design themes for supportive technologies for individuals in recovery from SUDs: 1) supporting twelve-step traditions and practices, 2) management of restlessness and moments of crisis, 3) agency and control over privacy and personal safety, 4) tracking progress and maintaining motivation in recovery, and 5) constructing a new normal. Below, we discuss each theme and provide examples of participant discussion and design artifacts.

\subsection{Supporting Twelve-Step Traditions and Practices}

Throughout the workshops, participants consistently sought ways to leverage technology to support and extend their participation in 12-step practices to support their recovery. Specifically, participants focused their designs on solving problematic logistical issues surrounding their engagement with 12-steps practices such as finding a sponsor and securing safe and affordable transportation to 12-step meetings. This focus on extending and streamlining engagement in 12step recovery was present in every workshop, but was especially prominent in the problem identification, ideation, and sketching workshops (i.e., workshops 4, 5, and 6). 
During the problem identification workshop, participants identified twenty-five prominent problems related to their day-to-day recovery, and then individually voted for the problems they found most personally relevant. Participants most often voted for problems related to traditional 12-step practices such as "Finding service opportunities," "Sponsor communication," and "Transportation to AA meetings" (Figure 1). In describing the challenge of finding local "same-day" service opportunities, a participant stated, "It's very difficult to find a unified list of service opportunities in the area. A list that is updated and could be filterable would be a godsend."

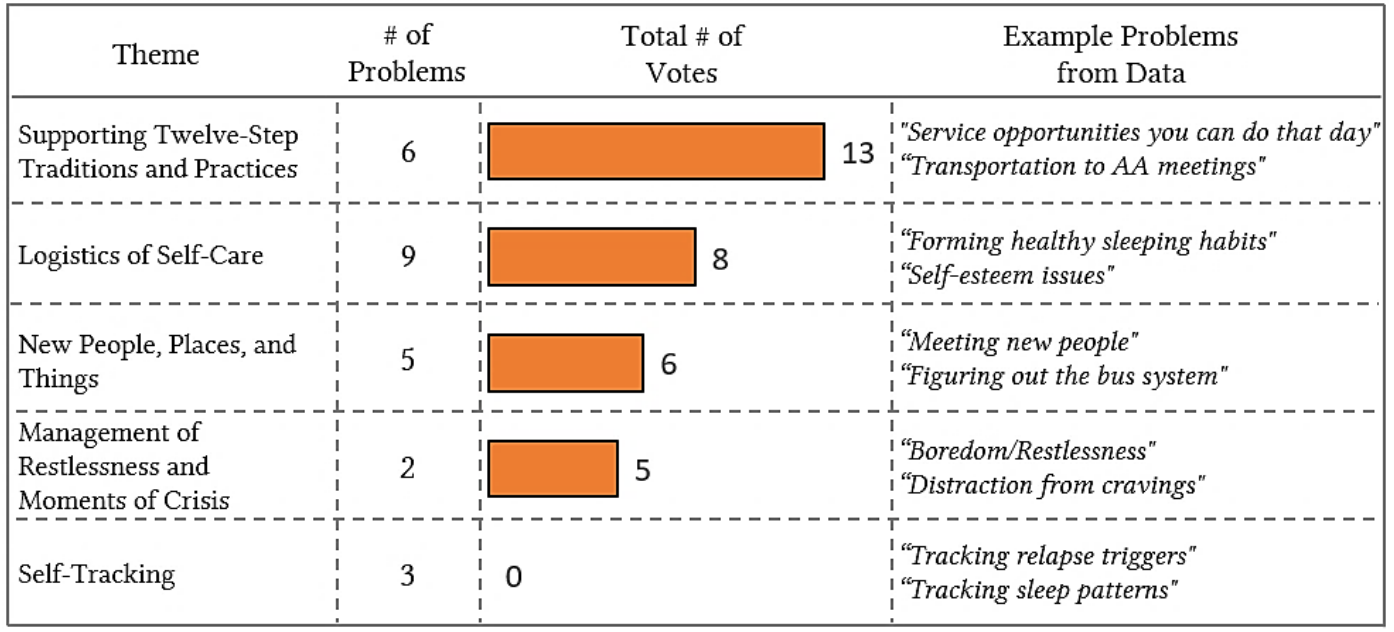

Fig. 1: Prevalence (i.e. number of problems) and personal relevance (i.e. total votes) of participant recovery related problems organized by design theme. Data were collected from workshop four in which participants identified twenty-five prominent problems related to their day-to-day recovery and then voted for the four problems they found most personally relevant.

Participants also focused on leveraging technology to support 12-step practices and traditions during the ideation workshop. Participants brainstormed fifty-four solutions to their most salient problems related to recovery and then individually voted for the most promising ideas (Figure 2). Among the thirty ideas that received the most votes, sixteen focused on providing support for 12step practices and traditions such as "Rideshare service for AA meetings" and "Searchable service work listings." Additionally, the idea that received the most participant votes was a smartphone application that would assist the user in searching for and communicating with a sponsor (Figure 2) - a core element of 12-step fellowships.

In the final workshop (i.e. sketching workshop), participants worked in groups of two and three to design and sketch technology to support their recovery. Each group was allowed to choose any idea developed in the previous workshop (i.e. ideation workshop), but independently, each group decided to focus on the same idea - the "Sponsor finder app." While this core app functionality was included in each team's app sketch, there was some diversity in terms of additional functionalities, features, and approaches. Participant sketches were comprised of four core themes focused on providing support for engagement with 12-step practices. First, participant sketches featured the ability to search for a potential sponsor based on individual characteristics (Figure 3a). These characteristics were diverse and specific to the individual. For instance, participants stated they wanted the ability to search for sponsors based on "experience," "length of sobriety," "spirituality," "personality and hobbies," "location," "expectations for sponsees," and "the way they work the 12-steps." Second, participants designed multiple ways for the user to schedule 
meetings and communicate with a potential or existing sponsor via text or voice communication (Figures 3b and 3c). Third, participants designed a "family tree" interface that would allow the sponsee to gain access and form connections with their sponsor's peer support network (Figure 3d). Finally, participant designs emphasized the importance of service work featuring functionalities to find local service work (Figures $4 \mathrm{a}$ and $\underline{4 b}$ ) and view completed service work (Figure 4c).

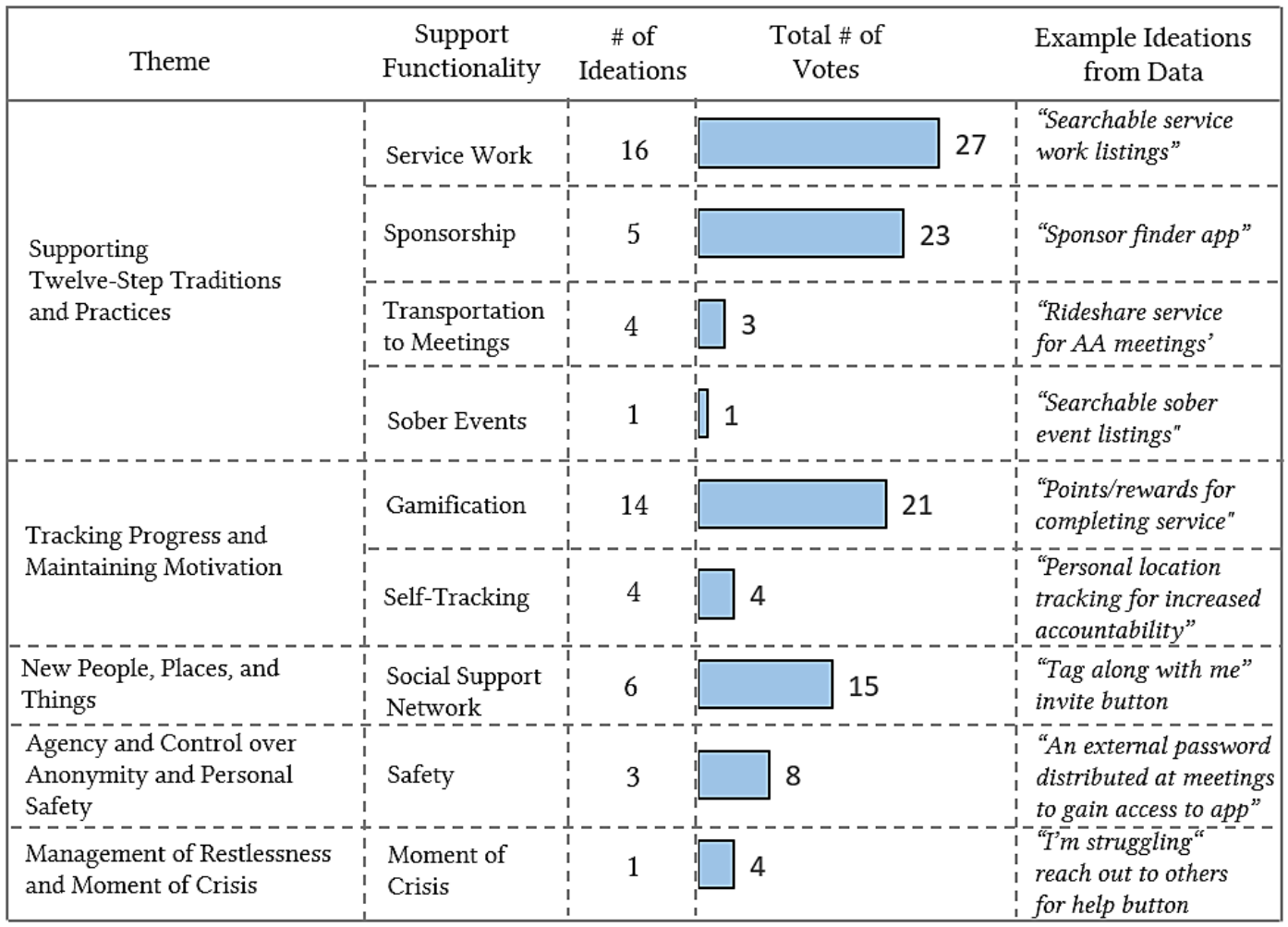

Fig 2: Prevalence (i.e. number of ideations) and popularity (i.e. total votes) of participant ideations organized by design theme and support functionality. Data were collected from workshop five in which participants brainstormed fifty-four solutions to their most salient problems related to recovery and then voted for the ideas showing the most promise.
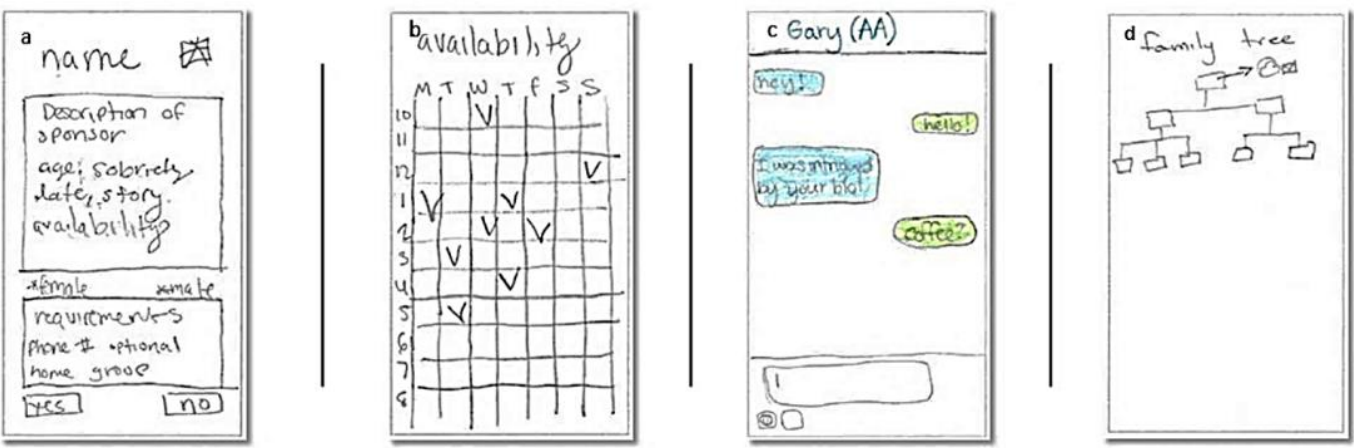

Fig. 3. Participant designs concerning a) finding a sponsor, b) scheduling meetings with a sponsor, c) communicating with a sponsor, and d) connection to a sponsor's support network. 

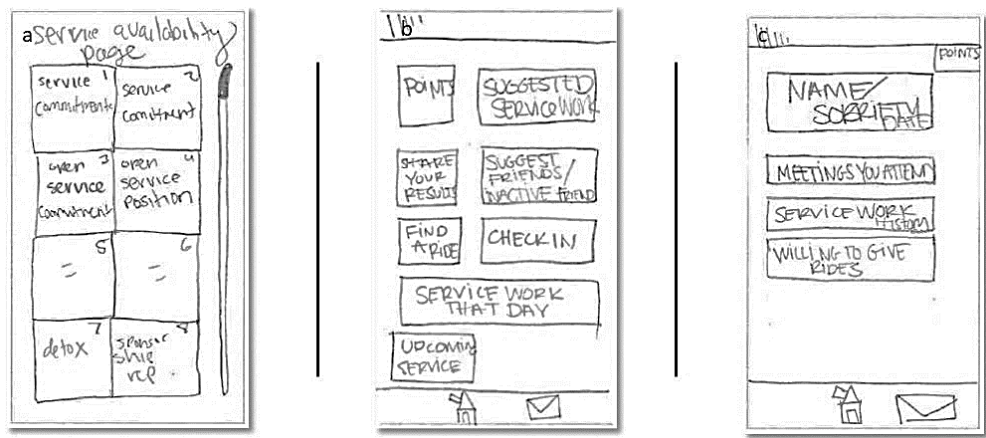

Fig. 4. Participant designs concerning a) finding service work, b) same-day service opportunities, and c) tracking service history.
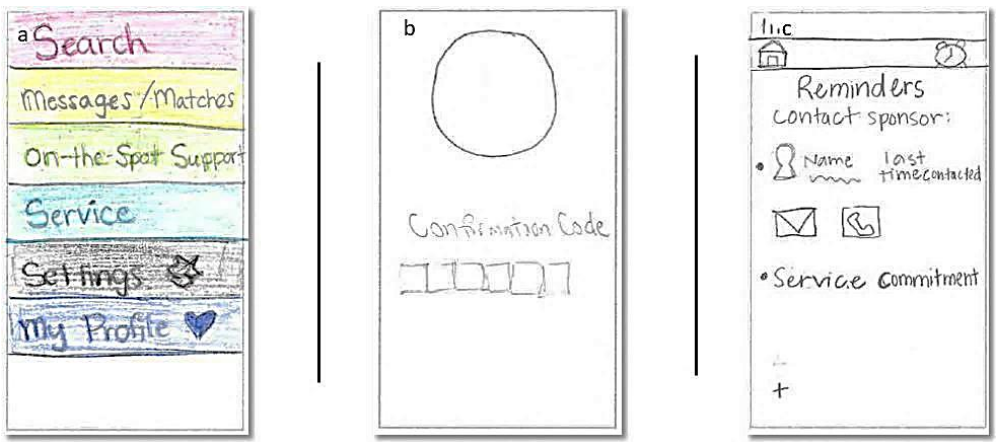

Fig. 5. Participant designs emphasizing need for a) on-the-spot support, b) user safety via password protected app access, and c) customized daily reminders

\subsection{Management of Restlessness and Moments of Crisis}

In addition to supporting the traditions and practices of 12-step fellowships, participants frequently focused on the dangers of restlessness and boredom in early recovery. During the first workshop, participants focused their discussion on several technologies that help them cope with these emotions. For instance, a participant stated she uses a low-tech strategy to mitigate restlessness.

"A simple strategy for me is coloring. I think it's good, it calms me down and makes me forget.

If I'm thinking way too much about things that are going on in my life and getting close to wanting to drink, then I just color and that makes me focus on that instead of everything else."

Another participant reported using Headspace, a mindfulness meditation app, to find focus during periods of restlessness: "The headspace app helps me meditate every day at the same time and makes it a habit... it helps with coaching me through the meditation and leaves little room for my mind to wander."

Restlessness is particularly dangerous for individuals in early recovery as it can precipitate emotional crises such as panic attacks and strong cravings-emotional states associated with relapse. Due to their unpredictable nature, several participants discussed the need for technology that would allow them to self-manage these crises - regardless of the time of day or situation in which they occur. This is especially vital since peer support, such as calling a sponsor or attending an AA meeting, may not always be readily available. Thus, it is important that individuals possess a consistently accessible self-management tool. For example, while exploring NoMo, a smartphone 
application designed for recovery, a participant said that she would use the "Guided breathing feature for panic attacks before they become unmanageable" or "if I felt an episode come on and I was say on the bus or something." However, participants also stated the simplicity of this feature might become dull or monotonous over prolonged use-thus limiting its effectiveness. In an attempt to create a more dynamic solution, a participant suggested the following feature.

"A thirty-minute timer activated when you have a craving. While the countdown is happening, it could give you things you can do like a coloring activity, games that are mindless and entertaining, or different options like a walk".

In addition to technology that would allow participants to self-manage crises, participants also brainstormed solutions that would instantly connect the user to their peer support network. For example, a participant suggested that supportive technology include an "I'm struggling button" that would instantaneously contact the user's support network - an idea that was well supported by other participants (Figure 2). A participant's sketch further developed this idea by implementing an "On-the-Spot Support" button that would immediately contact the user's sponsor and other preselected members of the user's support network via text message (Figure 5a).

In summary, participants highlighted the need for technology to get access to in-the-moment support either through self-management or through social support during dangerous periods of restlessness, panic attacks, and intense cravings.

\subsection{Agency and Control over Anonymity and Personal Safety}

Early recovery can be a particularly vulnerable stage in a person's life. Considerations for managing stigma, privacy, personal and group anonymity, trust, and personal safety were all recurrent themes for participants in our design workshops.

While interacting with currently available supportive technology designed for recovery, participants expressed concern and caution when confronted with the ability to share personal recovery details with unknown individuals in an online mutual support environment. For instance, a participant stated, "I don't like that there are random people viewing my comments and sober time. I don't like receiving messages from random people." She went on to say that she wanted "privacy to be the default setting," and that she would like the option of changing the privacy setting when she felt "ready" to share.

Other participants saw the potential benefits of an open online community, but only if properly aligned with 12-step traditions regarding anonymity. For instance, participants liked the open community structure found in KoKo, a social media platform that allows the user to anonymously help and receive help from users concerning issues related to stress, bullying, dating, etc. "If you use an algorithm to restrict the use of specific places (e.g., meeting groups) or names, it may work well for recovery. The fact that it is anonymous is cool."

In addition to concerns for personal anonymity, participants also mentioned the need to protect the anonymity and sanctity of local 12-step meetings. For example, a participant disliked the user-generated reviews of local AA and NA meetings she saw on Addicaid, a smartphone app designed for recovery.

"A comment might steer me from meetings I really like. A meeting is not like a restaurant on

Yelp. They should not be judged in that way. Each time a meeting is different. You should give it multiple tries."

Protecting personal safety while using technology for recovery was also of primary importance to participants. For example, while sketching her design of the "Sponsor Finder" smartphone application, a participant suggested the app include a mechanism to report "Creepy" 
behavior in order to remove " $13^{\text {th }}$ steppers"-individuals trying to initiate a sexual relationship with somebody new to recovery (which is viewed as a predatory practice in the community). Furthermore, many participants featured password protection in their sketches (Figure 5b). Participants suggested that app passwords be distributed during in-person AA meetings to help prevent individuals outside of recovery from using the app in a predatory manner. Physical attendance at a meeting was reported as a reasonable means for vetting online attendees as that real-world action allowed the development of trust and a mutual agreement of respecting others' anonymity and the group's primary purpose.

Participants were weary of using technology for recovery if it did not provide means for establishing trust or mutually agreed upon practices around personal anonymity, personal safety, group anonymity, and the primary purpose of a recovery venue. Thus, to gain the trust of the recovery community, technology must consider ways in which anonymity and safety practices could be established and continually reinforced through socio-technical means.

\subsection{Tracking Progress and Maintaining Motivation in Recovery}

Participant discussions and designs frequently focused on self-tracking and gamification functionalities to track progress and maintain motivation in recovery. Participants adopted opposing views regarding the appropriateness of these functionalities for the purposes of recovery. Some participants stated that self-tracking and gamification could assist individuals in staying accountable to their recovery goals. However, others expressed trepidation, stating that these functionalities trivialize the recovery process and undermine intrinsic motivation. Below, we review participant discussion and design artifacts regarding self-tracking and gamification technology for recovery.

5.4.1 Self-Tracking Recovery. Participants reacted positively to self-tracking functionalities while interacting with currently available technologies designed to support recovery (i.e., workshop \#2). For example, while interacting with WeConnect, a smartphone application designed for recovery, a participant stated, "I like the fact that it's a daily planner and you can track meetings, locations, daily chart of meetings so I can keep track of my 90 in 90 [ninety meetings in ninety days]." Another participant saw self-tracking as potentially helpful in coping with cross-addictions, "tracking your fill-in vices like drinking soda or smoking cigarettes....track how often you log on to your phone to procrastinate when you have a lot of things to do." Furthermore, participants stated that self-tracking provides a tangible recognition of their progress and accomplishments - a rare opportunity in recovery. For instance, a participant stated she uses journals to "track how I have changed over time." Another participant stated she uses daily checklist on her smartphone to "help me feel like I accomplished something today. That's important for when I get down on myself."

However, despite positive reactions to the self-tracking features they experienced in currently available technology, participants did not focus on self-tracking when asked to brainstorm solutions or sketch designs for their versions of supportive technology. For instance, participants generated four ideas that featured self-tracking; however, these ideas received a relatively low number of votes compared to other support functionalities (Figure 2). Participant sketches followed a similar pattern.

Finally, while some participants reacted positively to self-tracking, others felt self-tracking might exacerbate feelings of guilt and inadequacy during the recovery process. A participant stated that the money saving calculator featured in the NoMo app induced feelings of guilt regarding her past spending behavior. Participants also stated that self-tracking has the potential to exacerbate 
existing problems related to low self-esteem and perfectionism - two salient problems raised by participants during the problem identification workshop (Figure 1).

5.4.2 Gamification in Recovery. Analogous to how participants viewed the role of self-tracking in recovery, opinions regarding extrinsic motivational tools (e.g., gamification) were discordant. Some participants stated that gamification has great potential value for recovery, while others feared it would negatively influence their recovery. However, unlike self-tracking, participants featured gamification prominently in their brainstormed solutions (Figure 2) and design sketches. For example, participants suggested receiving points, trophies, or medals for engaging in service to the community, and featured said functionality prominently in their sketches (igures $4 \mathrm{~b}$ and $\underline{4 \mathrm{c}}$ ). Furthermore, a participant suggested that gamification could help individuals in recovery stay accountable. She stated that a potential user of the technology would "lose points if you say you were going somewhere but then don't show up without 24-hour notice or find a substitute."

Participants eschewed the idea of individual leaderboards, as they would break with the 12step tradition stating that all members are equal. Rather, participants suggested creating community leaderboards and sober house community competitions. Participants were also careful to ensure that gamification not be used to "shame people for relapsing." Therefore, rather than losing points for relapsing, participants suggested providing "amplified points for coming back" following relapse. When asked how points should be used, a participant suggested that users redeem points for prizes such as "luxuries we don't usually buy like nail polish, spa treatment, getting nails done."

Other participants raised concerns regarding the appropriateness of gamification for recovery. A participant stated that receiving points for recovery related activities "trivializes recovery." In addition to undermining intrinsic motivation, participants stated that gamification is "too similar to gambling" and may exacerbate underlying problems.

The potential roles of self-tracking and gamification in SUD recovery are both promising and risky. Given the split opinion of participants, supporting technologies should at the very least include an opportunity to opt out of these functionalities.

\subsection{Constructing a New Normal}

Participants, most of whom were new to recovery, described the inherent difficulties associated with navigating a novel substance-free lifestyle and fostering new relationships supportive of their sobriety.

5.5.1 Logistics of Self-Care. In beginning a new life in recovery, participants often spoke of their struggle towards incorporating a renewed focus on physical and mental health. Participants frequently spoke of their day-to-day difficulties in forming new healthy habits including personal hygiene, cleaning their living space, and eating healthy. For those unfamiliar with recovery, it may be challenging to view these activities as new or difficult. However, during the months or years of substance use, these beneficial daily routines often take a backseat, making it difficult to develop and sustain new healthy habits. For instance, a participant discussed the difficulties she has with self-care and suggested the following solution, "Having a checklist of things you need to do to care for yourself, like showering and grooming." Her design featured a daily reminder page that would allow the user to create custom alert prompts (Figure 5c). Additionally, participants also described problems they have with healthy eating and co-morbid eating disorders (Figure 1) and expressed a desire for technology to provide support toward consuming a sufficient amount of protein, avoiding purging after meals, and maintaining a healthy weight. 
In addition to physical self-care, participants identified multiple problems related to reshaping their sense of self and confronting deleterious emotions and mental states that precipitated and sustained their substance use in the past. For example, during the fourth workshop, participants identified problems such as "perfectionism," "self-esteem issues," "co-dependency," and "anxiety" as being inextricably linked to their substance use (igure 1). Participants stated that these harmful emotional states were key contributors to the onset of their substance abuse. Additionally, participants stated that they used substances to mask and cope with these emotions in the past and believe, if not properly confronted, will lead them to relapse in the future. To this point, a participant stated that she believes the key to her long-term recovery is to "learn more about myself and finding those triggers and just finding more about why I do the things that I do."

5.5.2 New People, Places, and Things. In addition to focusing on physical and mental health, participants spoke of difficulties in forming new "sober" relationships, finding reliable transportation, and securing employment supportive of their recovery. Participants highlighted the challenge and importance of forging supportive and nurturing relationships and identified "meeting new people," "relationship issues," and "sober dating" as prominent recovery challenges (Figure 1). During the ideation workshop, one of the most popular ideas was a smartphone application feature that would allow users to invite others in recovery to service events and sober activities they will be attending. Participants referred to this feature as a "Tag along with me" button (Figure 2). In addition to forming a social support network, participants focused on finding accessible and reliable transportation and securing employment. For example, a participant stated, "I am afraid of bus system and I don't have enough money for Uber. I would love to get a ride from other people in recovery going to the same meeting or running errands." Another participant detailed her struggle finding a job that "does not serve liquor and is a positive work environment for my recovery."

Participants expressed the importance and difficulty of navigating the major life changes associated with beginning the recovery process such as focusing on physical and mental health, finding transportation and employment, and developing and maintaining a social support network. Supportive technology that guides individuals through these difficult transitions could greatly assist the recovery process.

\section{DISCUSSION}

In this section, we use terminology outlined in Sas et al. [83] to present implications for the design of supportive technology for individuals in recovery from SUDs. In their research, Sas et al. [83] present a taxonomy to describe and categorize design implications within $\mathrm{HCI}$ including sensitizing concepts, abstractions, meta-abstractions, instantiations, and prescriptions. Below, we use this terminology to frame our design implications. Specifically, we present design implications in the form of one abstract functionality, one instantiation, and two sensitizing concepts.

\subsection{Abstract Functionality: Leveraging Existing Recovery Support Networks}

Throughout the participatory design workshops, participants focused on developing supportive technologies that would leverage 12-step traditions and practices to form additional socio-technical pathways toward peer support, mentorship, and assistance in times of need. As shown in the Supporting Twelve-Step Traditions and Practices section of our results, participants focused on developing technology to support their search for a 12-step sponsor to meet their individualized need for mentorship in recovery. Furthermore, as shown in the New People, Places and Things section of our results, participants described their need for a supportive peer network and 
suggested technologies for inviting others to AA meetings and service work opportunities. Finally, as outlined in the Management of Restlessness and Moments of Crisis section of our results, participants sketched an "On-the-Spot Support" feature that would immediately connect them to their 12-step sponsor and peer support network in times of need. In each of these examples, participants focused on developing tools that would empower them to leverage the robust peer support network made available through their engagement in 12-step fellowships.

The design emphasis on 12-step fellowships as a means to facilitate peer support was a participant-driven focus. Without participant input, we may have overlooked this viewpoint due to the sphere of controversy surrounding 12-step fellowships within scientific literature. For instance, many detractors of 12-step fellowships cite the core religious elements of the programlikening their practices to that of a cult rather than a treatment program [15]. Additionally, research findings concerning the effectiveness of 12-step fellowships have been divergent [48], with evidence of their effectiveness in treating SUDs $[46,89]$ appearing as frequently as evidence citing their ineffectiveness [30,56]. Furthermore, concerning the contexts in which 12-step practices are effective in treating SUDs, the underlying causal mechanisms contributing to their efficacy remain unclear and understudied. Thus, without sufficiently understanding the mechanisms of 12-step fellowships that contribute to their efficacy, there is no credible way to improve the treatment they offer-calling into question whether additional time and money should be invested into technologically extending them as a treatment.

In contrast to the controversy around 12-steps as a treatment, there is a considerably more favorable view of 12-steps as a source of social support. Our results indicate that 12-step fellowships were extremely important to participants in providing a robust and readily available social support network. Future design in this context should refrain from dismissing the impactful role of 12-step programs. Prior research indicates that peer support has been shown to be effective in facilitating SUD recovery, reducing relapse rates, and increasing retention in treatment programs [25,77]. Furthermore, Allen et al. [1] suggest that 12-step fellowships may leverage peer support more effectively than other psychosocial treatments, especially among individuals who lack a strong social support network. Thus, future design of technologies for SUD recovery should leverage the robust and readily available support network available through 12-step fellowships as a means of creating additional pathways for users to connect with peer support, mentorship, and assistance in times of need. This design recommendation represents a contrast to previous work in HCI regarding SUD recovery, where most technical solutions have focused on detecting and tracking substance use, $[47,95,100]$ and has not connected with 12-step programs and approaches.

While participants in this study focused on leveraging the social support aspects of 12-step fellowships, we believe this study serves as a template for future work investigating how technology can enhance and compliment other forms of long-term continuing care. 12-step fellowships are the most commonly used help setting in today's recovery landscape, however, that may change over time. Future work should continue to strive towards integrating technology as a complimentary source of support in commonly used long-term continuing care models.

\subsection{Instantiation: Sponsor App}

The "Sponsor Finder" app, developed by participants during the sketching workshop, is a concrete instantiation addressing the abstract functionality presented above (i.e., leveraging existing recovery support networks). We chose to focus on this specific instantiation due to its universally acknowledged potential among our participants and due to the participants' shared commitment 
toward its design. Particularly, among their fifty-four brainstormed ideas, participants recognized the "Sponsor Finder" app as the single most promising solution towards supporting their recovery. Additionally, when instructed to select a single ideation to sketch during the sixth workshop, each design team independently decided to work on the "Sponsor Finder" app.

Participant sketches of the "Sponsor Finder" app identify three explicit functionalities of this instantiation: 1) finding a sponsor, 2) supporting the logistics of working with a sponsor, and 3) leveraging a sponsor's contacts and support networks. Primarily, participants designed a customizable search functionality that enables an individual to find a sponsor to fit their personalized recovery needs (Figure 3a). Previous investigations into the benefits of sponsorship support this functionality. For instance, Bond et al. [12] found that having a sponsor was significantly and positively correlated with abstinence at one and three year follow-ups. In addition, having a sponsor predicted an increase in perceived personal and social support during recovery [81]. Furthermore, Tonigan et al. [90] suggested that acquiring a sponsor early in recovery (i.e., within first three months) leads to a higher probability of abstinence compared to acquiring a sponsor later in recovery (i.e., after 7 months into recovery). This finding indicates a potential timesensitive relationship between sponsorship and abstinence from alcohol. The "Sponsor Finder" app may expedite the search for a sponsor and allow users to acquire a sponsor earlier in their recovery, resulting in a relationship that is potentially more beneficial. Moreover, prior research indicates that sponsorship is also beneficial for the sponsor. Pagano et al. [72] found that individuals with an AUD who helped other individuals with an AUD were better able to maintain long-term sobriety.

In addition to the sponsor search functionality, participant sketches of the "Sponsor Finder" app focused on supporting the logistics of working with a sponsor. Participants designed technical features to schedule meetings, share recovery "homework" assignments, and communicate with their sponsor via text message. Improving the logistics of working with a sponsor may allow for more consistent sponsor/sponsee meetings. In support of this functionality, prior research indicates that increased contact with a sponsor is negatively associated with relapse [84].

Finally, participants designed a "family tree" interface that would allow the sponsee to gain access and form connections with their sponsor's peer support network (Figure 3d). This feature provides sponsees with a bridge to a robust peer support network available through their sponsor's existing recovery contacts. Sheeran [84] suggests that the most significant factor in successful recovery is the ability and willingness to "reach out to others for help." A larger peer support network may enable the sponsee to connect with a more diverse set of perspectives and experiences in recovery. Specifically, peers in recovery may share specific relevant experiences that their immediate sponsor may not, such as dealing with a divorce, issues related to childcare, or finding local sober-appropriate employment. Finally, a large peer network is more robust to changes in sponsorship that may result from a sponsor moving to a new area or relapsing (a relapse means that they are no longer eligible to sponsor). When a change occurs, a larger support network will provide the sponsee with additional peer support resources to close the gap in mentorship.

The "Sponsor Finder" app instantiation leverages existing recovery support networks to expedite and customize the search for mentorship, to enable consistent face-to-face meetings with a sponsor, and to connect with a robust support network via a sponsor's existing recovery contacts. 


\subsection{Sensitizing Concept: Sources of Motivation}

Twelve-step fellowships have often utilized non-technological forms of gamification and selftracking to motivate and mark progress in recovery. For example, members of AA receive milestone tokens representing the length of continuous sobriety. However, when discussing the role of digital gamification and self-tracking in SUD recovery, participants adopted opposing viewpoints on their appropriateness and usefulness. Below, we connect our findings to prior research that suggests that gamification and self-tracking may be harmful to some individuals. Finally, we provide implications for design focused on maximizing the benefit of these technologies while minimizing their potential for harm in this sensitive context.

6.3.1 Implications for gamification design. While some participants strongly focused on including elements of gamification in their designs for SUD recovery, others felt it stood in direct opposition of an intrinsically motivated recovery perspective. Prior research indicates there are measurable and relatively stable individual differences in the expression of intrinsic or extrinsic motivational orientations [3]. Thus, individual differences may determine the suitability of gamification for SUD recovery.

The opposing participant views concerning gamification are echoed within scientific literature. This debate has traditionally centered around the role of intrinsic motivation, which refers to doing something because it is inherently interesting or enjoyable, and extrinsic motivation, which refers to doing something because it leads to a separate external outcome [82]. Prior research has shown that extrinsic motivators, such as rewards or recognition, can induce positive short-term behavioral outcomes. Higgins and Bundy [43] showed that monetary rewards resulted in significantly higher rates of abstinence and increased participation in treatment compared to individuals who received standard therapy. Additionally, gamification techniques have shown early promise in increasing abstinence rates by reducing attentional bias modification in individuals in recovery from SUDs [17]. However, intrinsic motivation has been shown to lead to more positive long-term recovery outcomes compared to purely extrinsic motivators $[19,20]$. Furthermore, prior research indicates that extrinsic motivation has the potential to actively reduce an individual's existing intrinsic motivation $[23,40]$ - a potentially disastrous outcome concerning individuals in recovery. In response to this assertion, Amabile [2] suggests that in some contexts, extrinsic motivation can be combined synergistically with intrinsic motivation - especially when initial intrinsic motivation is high. Further research is required to distinguish the contexts in which gamification can be helpful from the contexts in which gamification can be dangerous for individuals in recovery.

Our results, in conjunction with prior research, suggest that gamification may be a helpful supplemental source of motivation for some individuals, and may be damaging for others. It is through that lens that we propose the following three design implications for gamification in SUD recovery. Primarily, due to the potentially damaging effects of gamification (e.g. reducing intrinsic motivation), we recommend that it be an optional feature in SUD recovery technology. Individuals should be able to opt-out of gamification features without precluding them from utilizing the full functionality of the supportive technology. Second, participants strongly suggested that gamification should avoid stigmatizing relapse. Therefore, we recommend that gamification reward progress without punishing or shaming moments of struggle in recovery (e.g. relapse). For example, the majority of individuals in long-term recovery will relapse at some point in their recovery [54]. Rather than punishing relapse through subtracting points or status within the gamification platform, the individual should be rewarded amplified points or status for coming 
back to the app and renewing their focus on recovery. Third, we recommend that social comparison and competition-based functionalities occur at the group level. Participants were opposed to the idea of individual leaderboards, as they would break with the 12-step tradition stating that all members are equal. Rather, we suggest the formation of SLE community competitions to track group accomplishments such as number of overall meetings attended or the number of hours engaged in service work. This recommendation is aligned with the core 12-step program tradition that individual members should refrain from seeking to place personal recovery accomplishments above the accomplishments of others.

6.3.2 Implications for self-tracking design. As identified by participants, self-tracking could be another external source of motivation for individuals in recovery. Self-tracking was controversial amongst our participants who felt that it could have both positive and negative impacts on their recovery. For example, participants felt that tracking the amount of money saved resulting from sobriety was misaligned with their perception of a successful recovery and could exacerbate feelings of guilt regarding past behavior. These concerns are echoed in previous studies that demonstrate the potential for self-tracking to have a negative impact on individuals with eating disorders [29], chronic health conditions [4], and women tracking their fertility [31]. Additionally, similar to our results, Kelley et al. [49] found that misaligned self-tracking goals negatively impacted the physical health and mental well-being of individuals with eating disorders.

Based on this prior research and our findings, we recommend that self-tracking technology for SUD recovery require individuals to work directly with a healthcare professional, therapist, recovery coach, or trusted sponsor to create personalized and appropriate self-tracking metrics and goals. Many individuals, especially those in early recovery, lack experience in defining and understanding "success" in recovery. This design recommendation will work to mitigate the potential for harm caused by self-tracking by ensuring individuals track meaningful and attainable goals aligned with their current needs.

\subsection{Sensitizing Concept: Safety in Recovery}

Throughout the participatory design workshops, participants consistently focused on protecting anonymity and ensuring the safety of individuals using digital technologies for recovery. This focus was most likely due to the persistent social disapproval and stigmatization associated with SUDs. Individuals with SUDs are frequently marginalized, often resulting in social exclusion during periods in which peer and familial support is vital for recovery [78]. Our participants, who were exclusively female, may have an increased motivation for anonymity given that prior research suggests that SUDs are more stigmatized in women than in men [14]. In addition to a heightened motivation for anonymity, the experience of our participants may have also resulted in an increased focus on ensuring personal safety in recovery. Prior research suggests that women with SUDs experience significantly higher rates of violence, victimization, and sexual assault compared to non-substance using women, and such experiences may contribute to increased substance use $[14,53,91,97]$. Therefore, to address the needs of women in recovery, supportive technology must align itself with the robust anonymity and safety expectations of its core users.

Indeed, anonymity in 12-steps has been investigated in the past. Rubya and Yarosh [80] found that individuals in recovery have a nuanced interpretation of anonymity. They suggest that individuals new to recovery tend to adopt a conventional interpretation of anonymity when interacting with online communities, equating anonymity to unidentifiability. However, they also found that as an individual's experience in recovery increases, their corresponding willingness to 
share personal details and desire to connect with others on a personal level increases. In other words, more experienced individuals are less likely to equate anonymity with strict unidentifiability. Rather, more experienced individuals tend to interpret anonymity as a social contract with other members (i.e., the mutual trust and implicit agreement for protecting each other's privacy). This social contract, and the mutual trust it entails, is a key tenant of physical inperson meetings that take place in 12-step fellowships. The social contract was established and is actively maintained in order to protect those seeking treatment from the stigma associated with SUDs [103].

Analogous to Rubya and Yarosh's findings [80], participants in this study (who were relatively new to recovery) sought unidentifiability in online communities and preferred technologies that ensured complete anonymity. However, during the ideation and sketching workshops (i.e. workshops 5 and 6), participants began to explore ways of incorporating the social contract, the element of physical meetings that simultaneously provides anonymity and the ability to share personal details, into their designs of supportive technology. For example, in their ideations and sketches of supportive technology, participants suggested that passwords be distributed during physical AA meetings to allow members of their in-person network to join their online community. Physical attendance at a meeting was reported as a reasonable means for vetting online attendees, as that real-world action reiterated the "social contract" of respecting others' anonymity and the group's primary purpose. Additionally, as previously mentioned, connecting with others through their sponsor's support network was seen by participants as a way to ensure the trustworthiness of new additions to their personal support network.

According to these findings, we recommend that future designs adopt a dynamic approach to ensuring safety and anonymity in recovery. Primarily, for individuals new to recovery (or individuals new to an online support community), tools should focus on instituting unidentifiable anonymity and limit the sharing of personal information. However, technology should also be flexible enough to support changes in the user's perspective of anonymity over time. Thus, technology should support the user's ability to build trust through the development of a sociotechnical version of a social contract within online communities. Participants in this study accomplished this by leveraging their in-person network-a strategy which future designs could adopt. Approaches that are more dynamic may also be used. For instance, future designs could empower individuals to share personal information as their number of online community interactions and trusted connections with other members increase over time.

\section{CONCLUSIONS}

SUDs are an increasingly widespread and dangerous public health issue facing our society. Technology designed for the needs and values of people in recovery may be able to supplement traditional treatment options, enhance long-term abstinence maintenance, and create new opportunities for social support. In this study, we conducted six participatory design workshops with women living in a sober living home to address three research questions aimed at aligning the design of supportive technology with the specific priorities, values, and challenges faced by individuals in early recovery from SUDs. In our first research question, we asked, "What specific challenges of early recovery may be ameliorated by supportive technologies?" To answer this question, we worked with participants to identify their most prominent day-to-day challenges in recovery. They indicated the most significant obstacles included problematic logistics surrounding 12-step participation, forming new "sober" relationships, managing maladaptive emotional states 
(e.g. restlessness, low self-esteem and anxiety), physical self-care, and maintaining anonymity while using supportive technology. Our second research questions asked, "What current recovery practices and behaviors may benefit most from supportive technologies?" To find the answer, we co-designed potential supportive technologies with participants. Their designs focused on tools that would empower them to leverage the robust peer support network made available through their engagement in 12-step fellowships, revealing the possible benefits that technologies may provide to 12-step practices. In our final research question, we asked, "What specific values and traditions of the recovery community should guide supportive technologies in this domain?" Through their ideations, sketches, and discussion, participants highlighted the importance of anonymity and safety in recovery. Additionally, participants focused on enhancing their intrinsic motivation and eschewed external sources of motivation that trivialized the recovery process, shamed relapse, or tracked metrics misaligned with recovery priorities. The overarching contribution of this work is in establishing specific recommendations and directions for supportive technologies for SUD recovery through a working partnership between technologists and people in active recovery.

\section{ACKNOWLEDGEMENTS}

We would like to thank the participants and SLE managers for their willingness to share their experience in recovery and for their expertise in designing supportive technology as well as the SLE managers for their help in organizing the workshop schedule. We also thank Dr. Amy Krentzman and members of the GroupLens labs for edits and suggestions. This study was funded by NSF grants 1651575 and 1464376.

\section{REFERENCES}

[1] J. Allen, R. F. Anton, T. F. Babor, J. Carbonari, K. M. Carroll, G. J. Connors, N. L. Cooney, F. K. Del Boca, C. C. DiClemente, and D. Donovan. 1998. Matching alcoholism treatments to client heterogeneity. Alcoholism: clinical and experimental research 22, 6 (1998), 1300-1311.

[2] Teresa M. Amabile. 1993. Motivational synergy: Toward new conceptualizations of intrinsic and extrinsic motivation in the workplace. Human resource management review 3, 3 (1993), 185-201.

[3] Teresa M. Amabile, Karl G. Hill, Beth A. Hennessey, and Elizabeth M. Tighe. 1994. The Work Preference Inventory: assessing intrinsic and extrinsic motivational orientations. Fournal of personality and social psychology 66, 5 (1994), 950.

[4] Jessica S. Ancker, Holly O. Witteman, Baria Hafeez, Thierry Provencher, Mary Van de Graaf, and Esther Wei. 2015. "You Get Reminded You're a Sick Person": Personal Data Tracking and Patients With Multiple Chronic Conditions. Fournal of Medical Internet Research 17, 8 (2015), e202. DOI:https://doi.org/10.2196/jmir.4209

[5] American Psychiatric Association. 2013. Diagnostic and statistical manual of mental disorders (DSM-5®). American Psychiatric Pub.

[6] Shiri Azenkot, Catherine Feng, and Maya Cakmak. 2016. Enabling Building Service Robots to Guide Blind People: A Participatory Design Approach. In The Eleventh ACM/IEEE International Conference on Human Robot Interaction (HRI '16), 3-10. Retrieved September 22, 2017 from http://dl.acm.org/citation.cfm?id=2906831.2906835

[7] Madeline Balaam, Stefan Rennick Egglestone, Geraldine Fitzpatrick, Tom Rodden, Ann-Marie Hughes, Anna Wilkinson, Thomas Nind, Lesley Axelrod, Eric Harris, Ian Ricketts, Susan Mawson, and Jane Burridge. 2011. Motivating Mobility: Designing for Lived Motivation in Stroke Rehabilitation. In Proceedings of the SIGCHI Conference on Human Factors in Computing Systems (CHI '11), 3073-3082. DOI:https://doi.org/10.1145/1978942.1979397

[8] Jakob E. Bardram, Mads Frost, Károly Szántó, Maria Faurholt-Jepsen, Maj Vinberg, and Lars Vedel Kessing. 2013. Designing Mobile Health Technology for Bipolar Disorder: A Field Trial of the Monarca System. In Proceedings of the SIGCHI Conference on Human Factors in Computing Systems (CHI '13), 2627-2636. DOI:https://doi.org/10.1145/2470654.2481364

[9] Jakob E. Bardram, Mads Frost, Károly Szántó, and Gabriela Marcu. 2012. The MONARCA Self-assessment System: A Persuasive Personal Monitoring System for Bipolar Patients. In Proceedings of the 2Nd ACM SIGHIT International Health Informatics Symposium (IHI '12), 21-30. DOI:https://doi.org/10.1145/2110363.2110370

[10] Janet C. Blodgett, Natalya C. Maisel, Ingrid L. Fuh, Paula L. Wilbourne, and John W. Finney. 2014. How effective is continuing care for substance use disorders? A meta-analytic review. Fournal of Substance Abuse Treatment 46, 2 (February 2014), 87-97. DOI:https://doi.org/10.1016/j.jsat.2013.08.022

[11] Thomas Bodenheimer, Kate Lorig, Halsted Holman, and Kevin Grumbach. 2002. Patient self-management of chronic disease in primary care. Fournal of the American Medical Association 288, 19 (2002), 2469-2475. 
[12] Jason Bond, Lee Ann Kaskutas, and Constance Weisner. 2003. The persistent influence of social networks and alcoholics anonymous on abstinence. fournal of studies on alcohol 64, 4 (2003), 579-588.

[13] Alan Borning and Michael Muller. 2012. Next Steps for Value Sensitive Design. In Proceedings of the SIGCHI Conference on Human Factors in Computing Systems (CHI '12), 1125-1134. DOI:https://doi.org/10.1145/2207676.2208560

[14] Kathleen T. Brady and Carrie L. Randall. 1999. Gender differences in substance use disorders. Psychiatric Clinics of North America 22, 2 (1999), 241-252.

[15] Charles Bufe. 1997. Alcoholics Anonymous: cult or cure? See Sharp Press.

[16] Kathleen M. Carroll, Samuel A. Ball, Steve Martino, Charla Nich, Theresa A. Babuscio, and Bruce J. Rounsaville. 2009. Enduring effects of a computer-assisted training program for cognitive behavioral therapy: a 6-month follow-up of CBT4CBT. Drug \& Alcohol Dependence 100, 1 (2009), 178-181.

[17] W. Miles Cox, Javad S. Fadardi, James M. Intriligator, and Eric Klinger. 2014. Attentional bias modification for addictive behaviors: clinical implications. CNS spectrums 19, 3 (2014), 215-224.

[18] Michael A. Cucciare, Eric A. Coleman, and Christine Timko. 2015. A conceptual model to facilitate transitions from primary care to specialty substance use disorder care: a review of the literature. Primary health care research \& development 16, 5 (2015), 492-505.

[19] John A. Cunningham, Linda C. Sobell, Mark B. Sobell, and Janet Gaskin. 1994. Alcohol and drug abusers' reasons for seeking treatment. Addictive Behaviors 19, 6 (1994), 691-696.

[20] Susan Curry, Edward H. Wagner, and Louis C. Grothaus. 1990. Intrinsic and extrinsic motivation for smoking cessation. Journal of consulting and clinical psychology 58, 3 (1990), 310.

[21] Jennifer L. Davidson and Carlos Jensen. 2013. Participatory design with older adults: an analysis of creativity in the design of mobile healthcare applications. In Proceedings of the 9th ACM Conference on Creativity \& Cognition, 114-123.

[22] Deborah A. Dawson, Risë B. Goldstein, and Bridget F. Grant. 2007. Rates and correlates of relapse among individuals in remission from DSM-IV alcohol dependence: a 3-year follow-up. Alcoholism: Clinical and Experimental Research 31, 12 (December 2007), 2036-2045. DOI:https://doi.org/10.1111/j.1530-0277.2007.00536.x

[23] Edward L. Deci, Richard Koestner, and Richard M. Ryan. 1999. A meta-analytic review of experiments examining the effects of extrinsic rewards on intrinsic motivation. Psychological bulletin 125, 6 (1999), 627.

[24] Victor Dibia. 2016. FOQUS: A Smartwatch Application for Individuals with ADHD and Mental Health Challenges. In Proceedings of the 18th International ACM SIGACCESS Conference on Computers and Accessibility (ASSETS '16), 311-312. DOI:https://doi.org/10.1145/2982142.2982207

[25] Patricia L. Dobkin, Mirella De Civita, Antonios Paraherakis, and Kathryn Gill. 2002. The role of functional social support in treatment retention and outcomes among outpatient adult substance abusers. Addiction 97, 3 (2002), 347-356.

[26] Gavin Doherty, David Coyle, and John Sharry. 2012. Engagement with Online Mental Health Interventions: An Exploratory Clinical Study of a Treatment for Depression. In Proceedings of the SIGCHI Conference on Human Factors in Computing Systems (CHI '12), 1421-1430. DOI:https://doi.org/10.1145/2207676.2208602

[27] Patrick L. Dulin, Vivian M. Gonzalez, Diane K. King, Danielle Giroux, and Samantha Bacon. 2013. Smartphone-Based, Self-Administered Intervention System for Alcohol Use Disorders: Theory and Empirical Evidence Basis. Alcohol Treat $Q$ 31, 3 (2013). DOI:https://doi.org/10.1080/07347324.2013.800425

[28] Pelle Ehn. 2008. Participation in design things. In Proceedings of the tenth anniversary conference on participatory design 2008, 92-101. Retrieved September 22, 2017 from http://dl.acm.org/citation.cfm?id=1795248

[29] Elizabeth V. Eikey and Madhu C. Reddy. 2017. It's Definitely Been a Journey: A Qualitative Study on How Women with Eating Disorders Use Weight Loss Apps. In Proceedings of the 2017 CHI Conference on Human Factors in Computing Systems, 642-654.

[30] Marica Ferri, Laura Amato, and Marina Davoli. 2006. Alcoholics Anonymous and other 12-step programmes for alcohol dependence. The Cochrane Library (2006).

[31] Mayara Costa Figueiredo, Clara Caldeira, Tera L. Reynolds, Sean Victory, Kai Zheng, and Yunan Chen. 2017. Self-Tracking for Fertility Care: Collaborative Support for a Highly Personalized Problem. PACM on Human-Computer Interaction 1, CSCW (December 2017), 36:1-36:21. DOI:https://doi.org/10.1145/3134671

[32] Lauren A. Fowler, Sidney L. Holt, and Deepti Joshi. 2016. Mobile technology-based interventions for adult users of alcohol: A systematic review of the literature. Addictive Behaviors 62, (November 2016), 25-34. DOI:https://doi.org/10.1016/j.addbeh.2016.06.008

[33] Peter D. Friedmann. 2013. Alcohol Use in Adults. New England fournal of Medicine 368, 4 (January 2013), 365-373. DOI:https://doi.org/10.1056/NEJMcp1204714

[34] Kathrin Gerling, Kieran Hicks, Michael Kalyn, Adam Evans, and Conor Linehan. 2016. Designing movement-based play with young people using powered wheelchairs. In Proceedings of the 2016 CHI Conference on Human Factors in Computing Systems, 4447-4458. Retrieved February 15, 2017 from http://dl.acm.org/citation.cfm?id=2858070

[35] Peter G. Gibson, Heather Powell, Amanda Wilson, Michael J. Abramson, P. Haywood, Adrian Bauman, Michael J. Hensley, E. Haydn Walters, and Jennifer JL Roberts. 2002. Self-management education and regular practitioner review for adults with asthma. Cochrane database of systematic reviews (2002). Retrieved September 22, 2017 from http://onlinelibrary.wiley.com/doi/10.1002/14651858.CD001117/full

[36] Bridget F. Grant, Risë B. Goldstein, Tulshi D. Saha, S. Patricia Chou, Jeesun Jung, Haitao Zhang, Roger P. Pickering, W. June Ruan, Sharon M. Smith, Boji Huang, and Deborah S. Hasin. 2015. Epidemiology of DSM-5 Alcohol Use Disorder. FAMA Psychiatry 72, 8 (August 2015), 757-766. DOI:https://doi.org/10.1001/jamapsychiatry.2015.0584

[37] Bridget F. Grant, Tulshi D. Saha, W. June Ruan, Risë B. Goldstein, S. Patricia Chou, Jeesun Jung, Haitao Zhang, Sharon M. Smith, Roger P. Pickering, Boji Huang, and Deborah S. Hasin. 2016. Epidemiology of DSM-5 Drug Use Disorder: Results From the National Epidemiologic Survey on Alcohol and Related Conditions-III. FAMA Psychiatry 73, 1 (January 2016), 39-47. DOI:https://doi.org/10.1001/jamapsychiatry.2015.2132 
[38] David H. Gustafson, Fiona M. McTavish, Ming-Yuan Chih, Amy K. Atwood, Roberta A. Johnson, Michael G. Boyle, Michael S. Levy, Hilary Driscoll, Steven M. Chisholm, and Lisa Dillenburg. 2014. A smartphone application to support recovery from alcoholism: a randomized clinical trial. FAMA psychiatry 71, 5 (2014), 566-572.

[39] David H. Gustafson, Bret R. Shaw, Andrew Isham, Timothy Baker, Michael G. Boyle, and Michael Levy. 2011. Explicating an evidence-based, theoretically informed, mobile technology-based system to improve outcomes for people in recovery for alcohol dependence. Substance use \& misuse 46, 1 (2011), 96-111.

[40] Michael D. Hanus and Jesse Fox. 2015. Assessing the effects of gamification in the classroom: A longitudinal study on intrinsic motivation, social comparison, satisfaction, effort, and academic performance. Computers \& Education 80, (2015), $152-161$.

[41] Deborah S. Hasin, Efrat Aharonovich, Ann O’leary, Eliana Greenstein, Martina Pavlicova, Srikesh Arunajadai, Rachel Waxman, Milton Wainberg, John Helzer, and Barbara Johnston. 2013. Reducing heavy drinking in HIV primary care: a randomized trial of brief intervention, with and without technological enhancement. Addiction 108, 7 (2013), 1230-1240.

[42] Holly Hedegaard, Margaret Warner, and Arialdi M. Miniño. 2017. Drug overdose deaths in the United States, 1999-2015. NCHS data brief 273 (2017), 1-8.

[43] Stephen T. Higgins and Alan J. Budney. 1993. Treatment of cocaine dependence through the principles of behavior analysis and behavioral pharmacology. NIDA research monograph 137, (1993), 97-97.

[44] A. Tom Horvath and Julie Yeterian. 2012. SMART Recovery: Self-empowering, science-based addiction recovery support. fournal of Groups in Addiction \& Recovery 7, 2-4 (2012), 102-117.

[45] Keith Humphreys. 1997. Clinicians' referral and matching of substance abuse patients to self-help groups after treatment. Psychiatric Services (1997)

[46] Keith Humphreys, Janet C. Blodgett, and Todd H. Wagner. 2014. Estimating the efficacy of Alcoholics Anonymous without self-selection bias: An instrumental variables re-analysis of randomized clinical trials. Alcoholism: Clinical and Experimental Research 38, 11 (2014), 2688-2694.

[47] Hsin-Liu (Cindy) Kao, Bo-Jhang Ho, Allan C. Lin, and Hao-Hua Chu. 2012. Phone-based Gait Analysis to Detect Alcohol Usage. In Proceedings of the 2012 ACM Conference on Ubiquitous Computing (UbiComp '12), 661-662. DOI:https://doi.org/10.1145/2370216.2370354

[48] Lee Ann Kaskutas. 2009. Alcoholics Anonymous effectiveness: Faith meets science. fournal of addictive diseases 28, 2 (2009), 145-157.

[49] Christina Kelley, Bongshin Lee, and Lauren Wilcox. 2017. Self-tracking for Mental Wellness: Understanding Expert Perspectives and Student Experiences. In Proceedings of the 2017 CHI Conference on Human Factors in Computing Systems 2017, (May 2017), 629-641. DOI:https://doi.org/10.1145/3025453.3025750

[50] John F. Kelly, Yifrah Kaminer, Christopher W. Kahler, Bettina Hoeppner, Julie Yeterian, Julie V. Cristello, and Christine Timko. A pilot randomized clinical trial testing integrated 12-Step facilitation (iTSF) treatment for adolescent substance use disorder. Addiction 112, 12 , 2155-2166. DOI:https://doi.org/10.1111/add.13920

[51] John F. Kelly and Rudolf Moos. 2003. Dropout from 12-step self-help groups: Prevalence, predictors, and counteracting treatment influences. Fournal of Substance Abuse Treatment 24, 3 (2003), 241-250.

[52] John F. Kelly, Julie Yeterian, and Mark G. Myers. 2008. TREATMENT STAFF REFERRALS, PARTICIPATION EXPECTATIONS, AND PERCEIVED BENEFITS AND BARRIERS TO ADOLESCENT INVOLVEMENT IN 12-STEP GROUPS. Alcohol Treat Q 26, 4 (2008). DOI:https://doi.org/10.1080/07347320802347053

[53] Dean G. Kilpatrick, Ron Acierno, Heidi S. Resnick, Benjamin E. Saunders, and Connie L. Best. 1997. A 2-year longitudinal analysis of the relationships between violent assault and substance use in women. fournal of consulting and clinical psychology 65, 5 (1997), 834 .

[54] Herbert D. Kleber, Roger D. Weiss, Raymond F. Anton, Tony P. George, Shelly F. Greenfield, Thomas R. Kosten, Charles P. O'Brien, Bruce J. Rounsaville, Eric C. Strain, Douglas M. Ziedonis, and others. 2007. Treatment of patients with substance use disorders, American Psychiatric Association. The American journal of psychiatry 164, 4 Suppl (2007), 5-123. Artie Konrad, Victoria Bellotti, Nicole Crenshaw, Simon Tucker, Les Nelson, Honglu Du, Peter Pirolli, and Steve Whittaker. 2015. Finding the adaptive sweet spot: Balancing compliance and achievement in automated stress reduction. In Proceedings of the 33rd Annual ACM Conference on Human Factors in Computing Systems, 3829-3838. Retrieved September 22, 2017 from http://dl.acm.org/citation.cfm?id=2702512

[56] R. J. Kownacki and W. R. Shadish. 1999. Does Alcoholics Anonymous work? The results from a meta-analysis of controlled experiments. Subst Use Misuse 34, 13 (November 1999), 1897-1916.

[57] Stephen Lindsay, Katie Brittain, Daniel Jackson, Cassim Ladha, Karim Ladha, and Patrick Olivier. 2012. Empathy, participatory design and people with dementia. In Proceedings of the SIGCHI Conference on Human Factors in Computing Systems, 521-530. Retrieved February 15, 2017 from http://dl.acm.org/citation.cfm?id=2207749

[58] Diana MacLean, Sonal Gupta, Anna Lembke, Christopher Manning, and Jeffrey Heer. 2015. Forum77: An analysis of an online health forum dedicated to addiction recovery. In Proceedings of the 18th ACM Conference on Computer Supported Cooperative Work \& Social Computing, 1511-1526. Retrieved May 16, 2017 from http://dl.acm.org/citation.cfm?id=2675146 Diana MacLean, Asta Roseway, and Mary Czerwinski. 2013. MoodWings: A Wearable Biofeedback Device for Real-time Stress Intervention. In Proceedings of the 6th International Conference on PErvasive Technologies Related to Assistive Environments (PETRA '13), 66:1-66:8. DOI:https://doi.org/10.1145/2504335.2504406

[60] Lena Mamykina and Elizabeth D. Mynatt. 2007. Investigating and Supporting Health Management Practices of Individuals with Diabetes. In Proceedings of the 1st ACM SIGMOBILE International Workshop on Systems and Networking Support for Healthcare and Assisted Living Environments (HealthNet '07), 49-54. DOI:https://doi.org/10.1145/1248054.1248068

[61] Mark Matthews and Gavin Doherty. 2011. In the Mood: Engaging Teenagers in Psychotherapy Using Mobile Phones. In Proceedings of the SIGCHI Conference on Human Factors in Computing Systems (CHI '11), 2947-2956. DOI:https://doi.org/10.1145/1978942.1979379 
[62] Mark Matthews, Stephen Voida, Saeed Abdullah, Gavin Doherty, Tanzeem Choudhury, Sangha Im, and Geri Gay. 2015. In Situ Design for Mental Illness: Considering the Pathology of Bipolar Disorder in mHealth Design. In Proceedings of the 17th International Conference on Human-Computer Interaction with Mobile Devices and Services (MobileHCI '15), 86-97. DOI:https://doi.org/10.1145/2785830.2785866

[63] R. Kathryn McHugh, Bridget A. Hearon, and Michael W. Otto. 2010. Cognitive-Behavioral Therapy for Substance Use Disorders. Psychiatric Clinics 33, 3 (September 2010), 511-525. DOI:https://doi.org/10.1016/j.psc.2010.04.012

[64] James R. McKay and Susanne Hiller-Sturmhöfel. 2011. Treating alcoholism as a chronic disease: approaches to long-term continuing care. Alcohol Research \& Health 33, 4 (2011), 356.

[65] A. Thomas McLellan, David C. Lewis, Charles P. O’brien, and Herbert D. Kleber. 2000. Drug dependence, a chronic medical illness: implications for treatment, insurance, and outcomes evaluation. Fama 284, 13 (2000), 1689-1695.

[66] David C. Mohr, Enid Montague, Colleen Stiles-Shields, Susan M. Kaiser, Christopher Brenner, Eric Carty-Fickes, Hannah Palac, and Jenna Duffecy. 2015. MedLink: a mobile intervention to address failure points in the treatment of depression in general medicine. In Proceedings of the 9th International Conference on Pervasive Computing Technologies for Healthcare, $100-107$.

[67] Rudolf H. Moos and Bernice S. Moos. 2006. Rates and predictors of relapse after natural and treated remission from alcohol use disorders. Addiction 101, 2 (February 2006), 212-222. DOI:https://doi.org/10.1111/j.1360-0443.2006.01310.x

[68] Michael J. Muller. 2003. Participatory design: the third space in HCI. Human-computer interaction: Development process 4235, (2003), 165-185.

[69] Michele A. Nealon-Woods, Joseph R. Ferrari, and Leonard A. Jason. 1995. Twelve-step program use among Oxford House residents: spirituality or social support in sobriety? fournal of Substance Abuse 7, 3 (1995), 311-318.

[70] Kathleen O'Leary, Arpita Bhattacharya, Sean A. Munson, Jacob O. Wobbrock, and Wanda Pratt. 2017. Design Opportunities for Mental Health Peer Support Technologies. In CSCW, 1470-1484.

[71] Judith S. Olson and Wendy A. Kellogg. 2014. Ways of Knowing in HCI. Springer Science \& Business.

[72] MARIA E. PAGANO, KAREN B. FRIEND, J. SCOTT TONIGAN, and ROBERT L. STOUT. 2004. Helping Other Alcoholics in Alcoholics Anonymous and Drinking Outcomes: Findings from Project MATCH. fournal of studies on alcohol 65, 6 (November 2004), 766-773.

[73] Pablo Paredes and Matthew Chan. 2011. CalmMeNow: Exploratory Research and Design of Stress Mitigating Mobile Interventions. In CHI '11 Extended Abstracts on Human Factors in Computing Systems (CHI EA '11), 1699-1704. DOI:https://doi.org/10.1145/1979742.1979831

[74] Pablo Paredes, Ran Gilad-Bachrach, Mary Czerwinski, Asta Roseway, Kael Rowan, and Javier Hernandez. 2014. PopTherapy: Coping with stress through pop-culture. In Proceedings of the 8th International Conference on Pervasive Computing Technologies for Healthcare, 109-117. Retrieved September 22, 2017 from http://dl.acm.org/citation.cfm?id=2686909

[75] Douglas L. Polcin and Diane Henderson. 2008. A Clean and Sober Place to Live: Philosophy, Structure, and Purported Therapeutic Factors in Sober Living Houses. Journal of psychoactive drugs 40, 2 (June 2008), 153-159.

[76] Steven L. Proctor and Philip L. Herschman. 2014. The continuing care model of substance use treatment: What works, and when is "enough,"“enough?" Psychiatry journal 2014, (2014).

[77] Sharon Reif, Lisa Braude, D. Russell Lyman, Richard H. Dougherty, Allen S. Daniels, Sushmita Shoma Ghose, Onaje Salim, and Miriam E. Delphin-Rittmon. 2014. Peer recovery support for individuals with substance use disorders: Assessing the evidence. Psychiatric Services 65, 7 (2014), 853-861.

[78] Robin Room. 2005. Stigma, social inequality and alcohol and drug use. Drug and Alcohol Review 24, 2 (March 2005), 143155. DOI:https://doi.org/10.1080/09595230500102434

[79] Sabirat Rubya and Svetlana Yarosh. 2017. Video-Mediated Peer Support in an Online Community for Recovery from Substance Use Disorders. In Proceedings of the 2017 ACM Conference on Computer Supported Cooperative Work and Social Computing (CSCW '17), 1454-1469. DOI:https://doi.org/10.1145/2998181.2998246

[80] Sabirat Rubya and Svetlana Yarosh. 2017. Interpretations of Online Anonymity in Alcoholics Anonymous and Narcotics Anonymous. PACM on Human-Computer Interaction 1, CSCW (December 2017), 91:1-91:22. DOI:https://doi.org/10.1145/3134726

[81] Mary M. Rush. 2002. Perceived social support: Dimensions of social interaction among sober female participants in Alcoholics Anonymous. Journal of the American Psychiatric Nurses Association 8, 4 (2002), 114-119.

[82] Richard M. Ryan and Edward L. Deci. 2000. Intrinsic and extrinsic motivations: Classic definitions and new directions. Contemporary educational psychology 25, 1 (2000), 54-67.

[83] Corina Sas, Steve Whittaker, Steven Dow, Jodi Forlizzi, and John Zimmerman. 2014. Generating implications for design through design research. In Proceedings of the SIGCHI Conference on Human Factors in Computing Systems, 1971-1980. Mary Sheeren. 1988. The relationship between relapse and involvement in alcoholics anonymous. fournal of Studies on Alcohol 49, 1 (1988), 104-106.

[85] Will Simm, Maria Angela Ferrario, Adrian Gradinar, Marcia Tavares Smith, Stephen Forshaw, Ian Smith, and Jon Whittle. 2016. Anxiety and Autism: Towards Personalized Digital Health. In Proceedings of the 2016 CHI Conference on Human Factors in Computing Systems (CHI '16), 1270-1281. DOI:https://doi.org/10.1145/2858036.2858259

[86] Valerie J. Slaymaker and Timothy Sheehan. 2008. The Impact of AA on Professional Treatment. In Recent Developments in Alcoholism. Springer, New York, NY, 59-70. DOI:https://doi.org/10.1007/978-0-387-77725-2_4

[87] Acar Tamersoy, Duen Horng Chau, and Munmun De Choudhury. 2017. Analysis of Smoking and Drinking Relapse in an Online Community. In Proceedings of the 2017 International Conference on Digital Health (DH '17), 33-42. DOI:https://doi.org/10.1145/3079452.3079463

[88] Acar Tamersoy, Munmun De Choudhury, and Duen Horng Chau. 2015. Characterizing Smoking and Drinking Abstinence from Social Media. In Proceedings of the 26th ACM Conference on Hypertext \& Social Media (HT '15), 139-148. DOI:https://doi.org/10.1145/2700171.2791247 
[89] Christine Timko, Anna DeBenedetti, and Rachel Billow. 2006. Intensive referral to 12-Step self-help groups and 6-month substance use disorder outcomes. Addiction 101, 5 (2006), 678-688.

[90] J. Scott Tonigan and Samara L. Rice. 2010. Is it beneficial to have an alcoholics anonymous sponsor? Psychology of Addictive Behaviors 24, 3 (2010), 397.

[91] Ellen Tuchman. 2010. Women and addiction: the importance of gender issues in substance abuse research. Fournal of addictive diseases 29,2 (2010), 127-138.

[92] Stephen Uzor, Lynne Baillie, and Dawn Skelton. 2012. Senior Designers: Empowering Seniors to Design Enjoyable Falls Rehabilitation Tools. In Proceedings of the SIGCHI Conference on Human Factors in Computing Systems (CHI '12), 11791188. DOI:https://doi.org/10.1145/2207676.2208568

[93] John Vines, Mark Blythe, Paul Dunphy, Vasillis Vlachokyriakos, Isaac Teece, Andrew Monk, and Patrick Olivier. 2012. Cheque Mates: Participatory Design of Digital Payments with Eighty Somethings. In Proceedings of the SIGCHI Conference on Human Factors in Computing Systems (CHI '12), 1189-1198. DOI:https://doi.org/10.1145/2207676.2208569

[94] Greg Wadley, Reeva Lederman, John Gleeson, and Mario Alvarez-Jimenez. 2013. Participatory Design of an Online Therapy for Youth Mental Health. In Proceedings of the 25th Australian Computer-Human Interaction Conference: Augmentation, Application, Innovation, Collaboration (OzCHI '13), 517-526. DOI:https://doi.org/10.1145/2541016.2541030

[95] Kuo-Cheng Wang, Yi-Hsuan Hsieh, Chi-Hsien Yen, Chuang-Wen You, Yen-Chang Chen, Ming-Chyi Huang, Seng-Yong Lau, Hsin-Liu (Cindy) Kao, and Hao-Hua Chu. 2014. SoberDiary: A Phone-based Support System for Assisting Recovery from Alcohol Dependence. In Proceedings of the 2014 ACM International foint Conference on Pervasive and Ubiquitous Computing: Adjunct Publication (UbiComp '14 Adjunct), 311-314. DOI:https://doi.org/10.1145/2638728.2638847

[96] Rina R. Wehbe, Diane K. Watson, Gustavo F. Tondello, Marim Ganaba, Melissa Stocco, Alvin Lee, and Lennart E. Nacke. 2016. ABOVE WATER: An Educational Game for Anxiety. In Proceedings of the 2016 Annual Symposium on ComputerHuman Interaction in Play Companion Extended Abstracts (CHI PLAY Companion '16), 79-84. DOI:https://doi.org/10.1145/2968120.2971804

[97] Sharon C. Wilsnack, Nancy D. Vogeltanz, Albert D. Klassen, and T. Robert Harris. 1997. Childhood sexual abuse and women's substance abuse: National survey findings. fournal of studies on alcohol 58, 3 (1997), 264-271.

[98] Svetlana Yarosh. 2013. Shifting Dynamics or Breaking Sacred Traditions?: The Role of Technology in Twelve-step Fellowships. In Proceedings of the SIGCHI Conference on Human Factors in Computing Systems (CHI '13), 3413-3422. DOI:https://doi.org/10.1145/2470654.2466468

[99] Svetlana Yarosh and Stephen Matthew Schueller. 2017. "Happiness Inventors": Informing Positive Computing Technologies Through Participatory Design With Children. fournal of medical Internet research 19, 1 (2017). Retrieved September 6, 2017 from https://www.ncbi.nlm.nih.gov/pmc/articles/PMC5285607/

[100] Chuang-Wen You, Ya-Fang Lin, Cheng-Yuan Li, Yu-Lun Tsai, Ming-Chyi Huang, Chao-Hui Lee, Hao-Chuan Wang, and Hao-Hua Chu. 2016. KeDiary: Using Mobile Phones to Assist Patients in Recovering from Drug Addiction. In Proceedings of the 2016 CHI Conference on Human Factors in Computing Systems (CHI '16), 5704-5709. DOI:https://doi.org/10.1145/2858036.2858185

[101] 2018. Mobile Fact Sheet. Pew Research Center: Internet, Science \& Tech. Retrieved June 23, 2018 from http://www.pewinternet.org/fact-sheet/mobile/

[102] Supplementary Materials | Surgeon General's Report on Alcohol, Drugs, and Health. Retrieved June 26, 2018 from https://addiction.surgeongeneral.gov/supplementary-materials

[103] Alcoholics Anonymous: Understanding Anonymity. Retrieved March 30, 2018 from https://www.aa.org/pages/en_US/understanding-anonymity

Received April 2018; revised July 2018; accepted September 2018. 